\title{
Relativistic corrections to the ground-state energy of the positronium molecule
}

\author{
Sergiy Bubin, ${ }^{1}$ Monika Stanke, ${ }^{1,2}$ Dariusz Kędziera, ${ }^{3}$ and Ludwik Adamowicz ${ }^{1,4}$ \\ ${ }^{1}$ Department of Chemistry, University of Arizona, Tucson, Arizona 85721, USA \\ ${ }^{2}$ Institute of Physics, Nicholas Copernicus University, ul. Grudziadzka 5, PL 87-100 Toruń, Poland \\ ${ }^{3}$ Department of Chemistry, Nicholas Copernicus University, ul. Gagarina 7, PL 87-100 Toruń, Poland \\ ${ }^{4}$ Department of Physics, University of Arizona, Tucson, Arizona 85721, USA
}

(Received 2 February 2007; published 11 June 2007)

\begin{abstract}
The leading-order relativistic corrections to the ground-state energy of the positronium molecule $\left(\mathrm{Ps}_{2}\right)$ have been computed within the framework of perturbation theory. As the zero-order wave function we used a highly accurate nonrelativistic variational expansion in terms of 6000 explicitly correlated Gaussians that yielded the lowest variational upper bound for this system to date. We also report some expectation values representing the properties of $\mathrm{Ps}_{2}$.
\end{abstract}

DOI: 10.1103/PhysRevA.75.062504

PACS number(s): 31.15.Pf, 31.30.Jv, 36.10.Dr

Positronium, Ps, is a system that consists of a positron and an electron. It is a lighter version of the hydrogen atom. However, due to the substantially smaller reduced mass, the Ps ground-state energy is only one-half of the hydrogen ground-state energy and its interparticle distance is 2 times the value in $\mathrm{H}$. Like the hydrogen atom, the energy, the wave function, and the expectation values of most operators representing physical properties of Ps can be determined exactly. These include the expectation values of relativistic corrections of the order of $\alpha^{2}$, where $\alpha=e^{2} / \hbar c$ is the fine structure constant. Those relativistic corrections, obtained as mean values of the corresponding relativistic operators with the exact Ps ground-state wave function,

$$
\psi=\frac{1}{\sqrt{8 \pi}} e^{-r_{1} / 2}
$$

(where $\mathbf{r}_{1}$ is the positron-electron distance) are

$$
\begin{gathered}
\left\langle\hat{H}_{\mathrm{MV}}\right\rangle=-5 / 64, \\
\left\langle\hat{H}_{\mathrm{D}}\right\rangle=1 / 8, \\
\left\langle\hat{H}_{\mathrm{OO}}\right\rangle=-1 / 8, \\
\left\langle\hat{H}_{\mathrm{SS}}\right\rangle= \begin{cases}1 / 4 & \text { for singlet, } \\
1 / 12 & \text { for triplet, }\end{cases} \\
\left\langle\hat{H}_{\mathrm{A}}\right\rangle= \begin{cases}0 & \text { for singlet, } \\
1 / 4 & \text { for triplet. }\end{cases}
\end{gathered}
$$

Here $\left\langle\hat{H}_{\mathrm{MV}}\right\rangle$ represents the mass-velocity term, $\left\langle\hat{H}_{\mathrm{D}}\right\rangle$ is the Darwin term, $\left\langle\hat{H}_{\mathrm{OO}}\right\rangle$ is the orbit-orbit term, and $\left\langle\hat{H}_{\mathrm{A}}\right\rangle$ is the annihilation channel term (the expressions for all of them will be given below). The two possible spin couplings of Ps, i.e., singlet and triplet, result in two different values for the energy of the spin-spin interaction terms $\left\langle\hat{H}_{\mathrm{SS}}\right\rangle$ and $\left\langle\hat{H}_{\mathrm{A}}\right\rangle$. With the above relativistic contributions and higher order QED corrections, the energy of Ps is known with a very high precision.

As was shown by Wheeler [1], it is possible to add an electron to Ps and form a stable Ps ${ }^{-}$anion. This was confirmed experimentally by Mills, who also measured the radiative decay rate for this system [2]. The binding energy of $\mathrm{Ps}^{-}$, which includes relativistic corrections, was determined with high precision by Bhatia and Drachman [3] and, more recently, by Drake and Grigorescu [4]. Ps ${ }^{-}$can be subsequently combined with another positron forming a neutral system, which in analogy to the hydrogen molecule can be called the positronium molecule or $\mathrm{Ps}_{2}$. The positronium molecule, though proven stable a long time ago by theoretical calculations [5], has not been directly observed in an experiment. Only recently an intense pulsed positron source was developed using a buffer gas trap to accumulate large number of positrons. This creates a dense plasma, which can be spatially focused and used for the formation of positronium molecules and positronium Bose-Einstein condensate $[6,7]$. This development may soon allow detection and experimental characterization of $\mathrm{Ps}_{2}$. This makes accurate calculations of this system more relevant. It should be mentioned that the $\mathrm{Ps}_{2}$ molecule is not a stable system with regard to electron-positron annihilation. However, calculations predict that its lifetime is almost 2 times as long as that of the positronium atom (in the singlet state) [8-14].

Apart from its experimental relevance, $\mathrm{Ps}_{2}$ is, and will remain, an interesting model system for theoretical calculations and analysis. Calculations can answer the question whether $\mathrm{Ps}_{2}$ is a genuine molecule similar to $\mathrm{H}_{2}$ with a covalent bond between the two positroniums, or it is a loosely bonded Ps cluster. Also, the calculations can very precisely determine the $\mathrm{Ps}_{2}$ dissociation energy and radiative lifetime.

Unlike the $\mathrm{H}_{2}$ molecule, the $\mathrm{Ps}_{2}$ molecule is a weakly bound system and so far only the existence of two bound states has been predicted with theoretical calculations. The ground state of $\mathrm{Ps}_{2}$ has zero orbital angular momentum, and both pairs of identical particles are in a singlet state. Recently, another bound state with orbital angular momentum $L=1$ and negative parity was predicted [15]. The ground state of the positronium molecule has been a subject of theoretical calculations for quite a long period of time (see, for example, [8-14,16-18] and references therein). This stable (if we disregard the annihilation process) electron-positron complex is interesting from many perspectives. It represents a fully nonadiabatic case of a hydrogenlike molecule and 
plays an important role in the general theory of the quantummechanical four-body problem. Much of the attention towards this system also comes from quantum electrodynamics (QED), because this is a purely leptonic system and because the electron-positron annihilation in $\mathrm{Ps}_{2}$ may proceed with the emission of zero, one, two, three, or more photons. The studies of the ground state of $\mathrm{Ps}_{2}$ so far have been mainly focused on obtaining a precise nonrelativistic binding energy and wave function, as well as some other properties such as the electron-positron contact density, which determines the radiative lifetime. At the same time, the methodology of very precise calculations on few-particle systems in recent years has advanced to the level where accurate treatment of relativistic and QED corrections becomes necessary. Very accurate calculations that include relativistic corrections have recently achieved a high level of precision for small atoms (see, for example, Refs. [19-23]) and, more recently, also for diatomic molecules $[24,25]$. However, the number of such studies done for positronic systems remains very limited. To our knowledge, the only high accuracy calculation of the relativistic effects for a positronic system that contains more than three particles was the work of Yan and Ho [26], where leading relativistic corrections for positronium hydride were computed.

In our previous work [14] we reported precise, the best to date, nonrelativistic energies and some other properties of the positronium molecule. Having generated a very accurate variational wave function, we now proceed to the evaluation of the relativistic corrections, the main goal of the present work. The calculations are performed within the framework of the Breit-Pauli formalism, as has been done for small atomic and molecular systems. As the calculations on the $\mathrm{He}$, $\mathrm{Li}$, and $\mathrm{Be}$ atoms show this formalism gives quite good results for light atomic systems. For such systems the relativistic effects are small, but not negligible, when such quantities as the ionization potentials, electron affinities, and transition energies are calculated.

In the Breit-Pauli formalism, a quantum system is described by the Hamiltonian that is a sum of the nonrelativistic Hamiltonian and a small relativistic correction,

$$
\hat{H}_{\text {tot }}=\hat{H}_{\text {nonrel }}+\alpha^{2} \hat{H}_{\text {rel }},
$$

where $\alpha$ is the fine structure constant. Due to the small magnitude of the relativistic correction it is possible to first solve the nonrelativistic problem and then account for the relativistic effects using the first-order perturbation theory. This is the approach used in this work.

The nonrelativistic Hamiltonian of an $N$-particle Coulomb system in the laboratory frame has the following form (in atomic units):

$$
\hat{H}_{\text {nonrel }}=-\sum_{i=1}^{N} \frac{1}{2 M_{i}} \nabla_{\mathbf{R}_{i}}^{2}+\sum_{i=1}^{N} \sum_{j>i}^{N} \frac{Q_{i} Q_{j}}{R_{i j}} .
$$

Here $\mathbf{R}_{i}, M_{i}, Q_{i}$ are the position, mass, and charge of the $i$ th particle; $\boldsymbol{\nabla}_{\mathbf{R}_{i}}$ is the gradient with respect to $\mathbf{R}_{i}$; and $R_{i j}=\mid \mathbf{R}_{j}$ $-\mathbf{R}_{i} \mid$ are interparticle distances. In the case of the positronium molecule all particle masses are equal to unity. We will also be assuming that particles 1 and 2 are positrons, and 3 and 4 are electrons, in which case $Q_{1}=1, Q_{2}=1, Q_{3}=-1$, and $Q_{4}=-1$.

Generally, $\hat{H}_{\text {rel }}$ consists of several contributions $[27,28]$,

$$
\hat{H}_{\mathrm{rel}}=\hat{H}_{\mathrm{MV}}+\hat{H}_{\mathrm{D}}+\hat{H}_{\mathrm{OO}}+\hat{H}_{\mathrm{SO}}+\hat{H}_{\mathrm{SS}}+\hat{H}_{\mathrm{A}} \text {, }
$$

that represent the mass-velocity (MV), Darwin (D), orbitorbit (OO), spin-orbit (SO), spin-spin (SS), and annihilation channel (A) corrections. In the case of $S$-symmetry (zero total angular momentum) of the zero-order (nonrelativistic) wave function, the expressions for certain terms in (5) become somewhat simplified and the relativistic operators then take the following forms:

$$
\begin{gathered}
\hat{H}_{\mathrm{MV}}=-\frac{1}{8} \sum_{i=1}^{N} \frac{1}{M_{i}^{3}} \nabla_{\mathbf{R}_{i}}^{4} \\
\hat{H}_{\mathrm{D}}=-\frac{\pi}{2} \sum_{i=1}^{N} \sum_{j \neq i}^{N} \frac{Q_{i} Q_{j}}{M_{i}^{2}} \delta\left(\mathbf{R}_{i j}\right), \\
\hat{H}_{\mathrm{OO}}=\frac{1}{2} \sum_{j=1}^{N} \sum_{i>j}^{N} \frac{Q_{i} Q_{j}}{M_{i} M_{j}} \frac{1}{R_{i j}}\left(\nabla_{\mathbf{R}_{i}^{\prime}}^{\prime} \nabla_{\mathbf{R}_{j}}+\frac{1}{R_{i j}^{2}} \mathbf{R}_{i j}^{\prime}\left(\mathbf{R}_{i j}^{\prime} \nabla_{\mathbf{R}_{i}}\right) \boldsymbol{\nabla}_{\mathbf{R}_{j}}\right), \\
\hat{H}_{\mathrm{SS}}=-\frac{8 \pi}{3} \sum_{i=1}^{N} \sum_{j>i}^{N} \frac{Q_{i} Q_{j}}{M_{i} M_{j}} \mathbf{S}_{i}^{\prime} \mathbf{S}_{j} \delta\left(\mathbf{R}_{i j}\right) \\
\hat{H}_{\mathrm{A}}=-2 \pi \sum_{i=1}^{N} \sum_{j>i}^{N} \frac{Q_{i} Q_{j}}{M_{i} M_{j}}\left(\frac{3}{4}+\mathbf{S}_{i}^{\prime} \mathbf{S}_{j}\right) \delta\left(\mathbf{R}_{i j}\right) .
\end{gathered}
$$

In the above expressions, $\delta\left(\mathbf{R}_{i j}\right)$ is the three-dimensional Dirac delta function, the prime (') represents the vector or matrix transposition, and $\mathbf{S}_{i}$ is the spin operator of the $i$ th particle.

The expressions for $\hat{H}_{\mathrm{MV}}, \hat{H}_{\mathrm{OO}}, \hat{H}_{\mathrm{SO}}$, and $\hat{H}_{\mathrm{SS}}$ are valid for any type of particles (if we disregard magnetic moment anomalies), while the expressions for $\hat{H}_{\mathrm{D}}$ and $\hat{H}_{\mathrm{A}}$ are given with the assumption that all particles in the system are spin$1 / 2$ fermions. It should also be emphasized that in the expression for $\hat{H}_{\mathrm{A}}$ the summation is only over the particleantiparticle pairs (such as $e^{+} e^{-}$).

In order to separate out the motion of the center of mass we use a set of internal coordinates as described in [29,30],

$$
\begin{gathered}
\mathbf{r}_{1}=-\mathbf{R}_{1}+\mathbf{R}_{2}, \\
\mathbf{r}_{2}=-\mathbf{R}_{1}+\mathbf{R}_{3}, \\
\cdots, \\
\mathbf{r}_{n}=-\mathbf{R}_{1}+\mathbf{R}_{N} .
\end{gathered}
$$

The coordinate transformation given by (12) sets particle 
1 in the origin of the new, internal coordinate frame. By using this set of internal coordinates we reduce the $N$-particle problem to an $n$-pseudoparticle problem with $n=N-1$.

In the internal coordinates the nonrelativistic Hamiltonian (4) has the following form:

$$
\begin{aligned}
\hat{H}_{\text {nonrel }}= & -\frac{1}{2}\left(\sum_{i=1}^{n} \frac{1}{\mu_{i}} \nabla_{\mathbf{r}_{i}}^{2}+\sum_{i=1}^{n} \sum_{j \neq i}^{n} \frac{1}{m_{0}} \nabla_{\mathbf{r}_{i}}^{\prime} \nabla_{\mathbf{r}_{j}}\right)+\sum_{i=1}^{n} \frac{q_{0} q_{i}}{r_{i}} \\
& +\sum_{i<j}^{n} \frac{q_{i} q_{j}}{r_{i j}}
\end{aligned}
$$

where $r_{i j}=\left|\mathbf{r}_{i}-\mathbf{r}_{j}\right|$. The masses and charges of the pseudoparticles are defined as $\mu_{i}=m_{0} m_{i} /\left(m_{0}+m_{i}\right)$ and $q_{i}=Q_{i+1}$, respectively. We also used the notation $m_{i}=M_{i+1}$. An analysis of the nonrelativistic Hamiltonian (13) shows that for $\mathrm{Ps}_{2}$ it represents a motion of three pseudoparticles around the charge of the reference particle placed in the center of the coordinate system. The Hamiltonian is isotropic (fully spherically symmetric) and its eigenfunctions should form a functional basis for irreducible representations of the fully spherically symmetric group of rotations. In particular, the ground state should be described by a spherically symmetric function with the $S$-symmetry. Due to isotropy of the Hamiltonian, it commutes both with the operator representing the square of the total angular momentum operator, and its $z$ component. Furthermore, since the Hamiltonian does not depend on spin, it also commutes with the square of the total spin operator, as well as with its $z$ component. Since the rotational motion of the positrons is strongly coupled to the rotational motion of the electrons, it is not possible to separate out the positron rotation as it is possible for $\mathrm{H}_{2}$, where the rotation of the molecular frame formed by the protons can be approximately separated from the internal motion of the electrons.

It can be shown (see Refs. [31,32]) that in the internal frame the terms included in $\hat{H}_{\text {rel }}$ have the following form:

$$
\begin{gathered}
\hat{H}_{\mathrm{MV}}=-\frac{1}{8}\left[\frac{1}{m_{0}^{3}}\left(\sum_{i=1}^{n} \boldsymbol{\nabla}_{\mathbf{r}_{i}}\right)^{4}+\sum_{i=1}^{n} \frac{1}{m_{i}^{3}} \boldsymbol{\nabla}_{\mathbf{r}_{i}}^{4}\right], \\
\hat{H}_{\mathrm{D}}=-\frac{\pi}{2}\left[\sum_{i=1}^{n}\left(\frac{1}{m_{0}^{2}}+\frac{1}{m_{i}^{2}}\right) q_{0} q_{i} \delta\left(\mathbf{r}_{i}\right)+\sum_{i=1}^{n} \sum_{j \neq i}^{n} \frac{1}{m_{i}^{2}} q_{i} q_{j} \delta\left(\mathbf{r}_{i j}\right)\right],
\end{gathered}
$$

$$
\begin{aligned}
\hat{H}_{\mathrm{OO}}= & -\frac{1}{2} \sum_{i=1}^{n} \sum_{j=1}^{n} \frac{q_{0} q_{j}}{m_{0} m_{j}}\left(\frac{1}{r_{j}} \boldsymbol{\nabla}_{\mathbf{r}_{i}}^{\prime} \nabla_{\mathbf{r}_{j}}+\frac{1}{r_{j}^{3}} \mathbf{r}_{j}^{\prime}\left(\mathbf{r}_{j}^{\prime} \boldsymbol{\nabla}_{\mathbf{r}_{i}}\right) \boldsymbol{\nabla}_{\mathbf{r}_{j}}\right) \\
& +\frac{1}{2} \sum_{i=1}^{n} \sum_{j>i}^{n} \frac{q_{i} q_{j}}{m_{i} m_{j}}\left(\frac{1}{r_{i j}} \boldsymbol{\nabla}_{\mathbf{r}_{i}}^{\prime} \boldsymbol{\nabla}_{\mathbf{r}_{j}}+\frac{1}{r_{i j}^{3}} \mathbf{r}_{i j}^{\prime}\left(\mathbf{r}_{i j}^{\prime} \boldsymbol{\nabla}_{\mathbf{r}_{i}}\right) \boldsymbol{\nabla}_{\mathbf{r}_{j}}\right),
\end{aligned}
$$

$$
\hat{H}_{\mathrm{SS}}=-\frac{8 \pi}{3} \sum_{i=1}^{n} \frac{q_{0} q_{i}}{m_{0} m_{i}} \mathbf{s}_{0}^{\prime} \mathbf{s}_{i} \delta\left(\mathbf{r}_{i}\right)-\frac{8 \pi}{3} \sum_{i=1}^{n} \sum_{j>i}^{n} \frac{q_{i} q_{j}}{m_{i} m_{j}} \mathbf{s}_{i}^{\prime} \mathbf{s}_{j} \delta\left(\mathbf{r}_{i j}\right),
$$

$$
\begin{aligned}
\hat{H}_{\mathrm{A}}= & -2 \pi \sum_{\substack{i=1 \\
x \bar{x} \text { pairs }}}^{n} \frac{q_{0} q_{i}}{m_{0} m_{i}}\left(\frac{3}{4}+\mathbf{s}_{0}^{\prime} \mathbf{s}_{i}\right) \delta\left(\mathbf{r}_{i}\right) \\
& -2 \pi \sum_{i=1}^{n} \sum_{\substack{j>i \\
x \bar{x} \text { pairs }}}^{n} \frac{q_{i} q_{j}}{m_{i} m_{j}}\left(\frac{3}{4}+\mathbf{s}_{i}^{\prime} \mathbf{s}_{j}\right) \delta\left(\mathbf{r}_{i j}\right) .
\end{aligned}
$$

In the above expressions $\mathbf{s}_{i} \equiv \mathbf{S}_{i+1}$.

The spin part of the positronium molecule ground-state wave function is

$$
\chi=\frac{1}{2}[\alpha(1) \beta(2)-\beta(1) \alpha(2)][\alpha(3) \beta(4)-\beta(3) \alpha(4)] .
$$

By expanding and rearranging the expression (19) in terms of eigenfunctions of the $\left(\mathbf{S}_{i}+\mathbf{S}_{j}\right)^{2}$ operators, one will find that the expectation values of $\mathbf{S}_{i}^{\prime} \mathbf{S}_{j}$ are equal to $-3 / 4$ in the case of a positron-positron or an electron-electron pair and zero in the case of a positron-electron pair.

In order to solve the nonrelativistic problem with the Hamiltonian (13) we used the variational method and expanded the wave function in terms of explicitly correlated Gausssian functions that have the following form:

$$
f_{k}=\exp \left[-\mathbf{r}^{\prime}\left(A_{k} \otimes I_{3}\right) \mathbf{r}\right]=\exp \left[-\mathbf{r}^{\prime}\left(L_{k} L_{k}^{\prime} \otimes I_{3}\right) \mathbf{r}\right] .
$$

In the above expression, $\mathbf{r}$ is a $3 n$-component position vector,

$$
\mathbf{r}=\left[\begin{array}{l}
\mathbf{r}_{1} \\
\mathbf{r}_{2} \\
\mathbf{r}_{3}
\end{array}\right],
$$

$A_{k}$ is a symmetric, positive definite $n \times n$ matrix of exponential parameters that are unique for each basis function, $I_{3}$ is $3 \times 3$ identity matrix, and $\otimes$ denotes the Kronecker product. For computational convenience, matrix $A_{k}$ is represented in the Cholesky-factored form as $L_{k} L_{k}^{\prime}$. In such a representation there are no constraints on the values of the elements of $L_{k}$, while elements of the original $A_{k}$ matrix must obey certain constraints to maintain its positive definiteness.

To account for the permutational and charge conjugation symmetry of the ground-state wave function of the positronium molecule, we need to apply the following projection operator to the basis functions (20):

$$
\left(1+\hat{P}_{13} \hat{P}_{24}\right)\left(1+\hat{P}_{12}\right)\left(1+\hat{P}_{34}\right),
$$

where $\hat{P}_{i j}$ stands for the permutation of particles $i$ and $j$. The result of applying the $\hat{P}_{i j}$ operators to the basis functions is equivalent to certain transformations of matrices $L_{k}$ that can be easily implemented in computer code. The symmetry of the $\mathrm{Ps}_{2}$ molecule has been discussed before by Kinghorn and Poshusta [16] and by Schrader [33,34].

In our calculations we used several basis sets of functions (20) obtained in our previous work on the positronium molecule [14]. The largest basis set in that prior work consisted of 5000 functions. In the present study, we followed the method of generating and optimizing the basis functions described in [14], and we increased the number of basis func- 
TABLE I. Expectation values of various operators for the ground state of $\mathrm{Ps}_{2}$ molecule computed with different basis sets in comparison with the same expectation values for two separated Ps atoms. Case (a) corresponds to the situation when both Ps atoms are in singlet state, while case (b) is when both are in triplet state. All numbers are in hartrees.

\begin{tabular}{lccccccc}
\hline \hline Basis size & $\left\langle H_{\text {nonrel }}\right\rangle$ & $\left\langle H_{\mathrm{MV}}\right\rangle$ & $\left\langle H_{\mathrm{D}}\right\rangle$ & $\left\langle H_{\mathrm{OO}}\right\rangle$ & $\left\langle H_{\mathrm{SS}}\right\rangle$ & $\left\langle H_{\mathrm{A}}\right\rangle$ & $\left\langle H_{\text {nonrel }}+\alpha^{2} H_{\text {rel }}\right\rangle$ \\
\hline 1000 & -0.516003787849 & -0.17182668 & 0.27398527 & -0.25294619 & $7.86716 \times 10^{-3}$ & 0.41687828 & -0.515989199222 \\
2000 & -0.516003789829 & -0.17183022 & 0.27399394 & -0.25294620 & $7.86569 \times 10^{-3}$ & 0.41689018 & -0.515989200374 \\
3000 & -0.516003790168 & -0.17183138 & 0.27399733 & -0.25294620 & $7.86403 \times 10^{-3}$ & 0.41689402 & -0.515989200478 \\
4000 & -0.516003790332 & -0.17183701 & 0.27400780 & -0.25294620 & $7.86403 \times 10^{-3}$ & 0.41690972 & -0.515989199550 \\
5000 & -0.516003790416 & -0.17183697 & 0.27400787 & -0.25294620 & $7.86401 \times 10^{-3}$ & 0.41690981 & -0.515989199624 \\
6000 & -0.516003790455 & -0.17183704 & 0.27400797 & -0.25294620 & $7.86397 \times 10^{-3}$ & 0.41690993 & -0.515989199656 \\
2 Ps (a) & -0.5 & -0.15625 & 0.25 & -0.25 & -0.5 & 0.0 & -0.500034946201 \\
2 Ps (b) & -0.5 & -0.15625 & 0.25 & -0.25 & 0.16666667 & 0.5 & -0.499999445298 \\
\hline \hline
\end{tabular}

tions to 6000. With that, the previous upper bound to the nonrelativistic ground-state energy of the positronium molecule was further improved. With 6000 explicitly correlated Gaussians we obtained the nonrelativistic energy value of -0.516003790455 hartree, which, we believe, is converged to about 1 part in $10^{10}$.

Having obtained the accurate nonrelativistic wave function we computed expectation values of the operators that constitute $\hat{H}_{\text {rel }}$. The expressions for matrix elements of those operators with basis functions (20), except for $\hat{H}_{\mathrm{OO}}$, can be found in [31]. In fact, in [31] we presented the derivations for a more general case of explicitly correlated Gaussians containing premultipliers in the form of even powers of $r_{1}$. The formulas for matrix elements of $\hat{H}_{\mathrm{OO}}$ with functions (20) are given in the Appendix. In Table I we show the convergence of the expectation values of operators (14)-(18) with increasing number of basis functions. As was mentioned above, the basis sets smaller than 6000 functions were taken from our previous calculations [14]. In Table I we also show the total nonrelativistic and relativistic energies obtained with different basis sets, as well as the results for the Ps atom for comparison.

In Table II we list expectation values of the interparticle distances and the delta functions that depend on the interpar-

TABLE II. Nonrelativistic expectation values of the interparticle distances, their squares, and contact densities computed with the largest basis of 6000 Gaussians used in this work. All numbers are in atomic units, i.e., distances are in bohrs, two-particle delta functions are in bohrs ${ }^{-3}$, etc.

\begin{tabular}{lc}
\hline \hline Quantity & Value \\
\hline$\left\langle r_{e^{+} e^{+}}\right\rangle$ & 6.033210260 \\
$\left\langle r_{e^{+} e^{-}}\right\rangle$ & 4.487154601 \\
$\left\langle r_{e^{+}}^{2}\right\rangle$ & 46.37487912 \\
$\left\langle r_{e^{+}}^{2}\right\rangle$ & 29.11270462 \\
$\left\langle\delta_{e^{+} e^{+}}\right\rangle$ & $6.2579505 \times 10^{-4}$ \\
$\left\langle\delta_{e^{+} e^{-}}\right\rangle$ & $2.2117759 \times 10^{-2}$ \\
$\left\langle\delta_{e^{+} e^{+} e^{-}}\right\rangle$ & $9.10802 \times 10^{-5}$ \\
$\left\langle\delta_{e^{+} e^{+} e^{-} e^{-}}\right\rangle$ & $4.56149 \times 10^{-6}$ \\
\hline \hline
\end{tabular}

ticle distances. The values have been computed with our nonrelativistic 6000-term wave function. Average interparticle distances calculated for smaller basis sets were already presented in [14]. In Table II we used the following shorthand notations for the expectation values of the delta functions: $\left\langle\delta_{e^{+} e^{-}}\right\rangle \equiv\left\langle\delta\left(\mathbf{r}_{e^{+}}-\mathbf{r}_{e^{-}}\right)\right\rangle, \quad\left\langle\delta_{e^{+} e^{+} e^{-}}\right\rangle \equiv\left\langle\delta\left(\mathbf{r}_{e^{+}}-\mathbf{r}_{e^{+}}\right) \delta\left(\mathbf{r}_{e^{+}}\right.\right.$ $\left.\left.-\mathbf{r}_{e^{-}}\right)\right\rangle,\left\langle\delta_{e^{+} e^{+} e^{-} e^{-}}\right\rangle \equiv\left\langle\delta\left(\mathbf{r}_{e^{+}}-\mathbf{r}_{e^{+}}\right) \delta\left(\mathbf{r}_{e^{+}}-\mathbf{r}_{e^{-}}\right) \delta\left(\mathbf{r}_{e^{-}}-\mathbf{r}_{e^{-}}\right)\right\rangle$.

Closer examination of the results for $\mathrm{Ps}_{2}$ in Tables I and II reveals that the convergence of the expectation values for the operators containing singularities (such as $\hat{H}_{\mathrm{MV}}, \hat{H}_{\mathrm{D}}, \hat{H}_{\mathrm{SS}}$, and $\left.\hat{H}_{\mathrm{A}}\right)$ is significantly worse than for those that do not have them $\left(\hat{H}_{\text {nonrel }}, \hat{H}_{\mathrm{OO}}\right.$, interparticle distances $)$. This behavior does not come as a surprise since the Gaussian basis functions are known for not satisfying the interparticle cusp conditions.

The interparticle distances allow insight into the structure of the $\mathrm{Ps}_{2}$ molecule. Although only two distinct average distances, the positron-positron distance (equal to the electronelectron distance) and the positron-electron distance, can be determined, they show that the structure of $\mathrm{Ps}_{2}$ is very different from the $\mathrm{H}_{2}$ molecule. While in $\mathrm{H}_{2}$ the average distance between the protons is about $1.4 \mathrm{bohr}$, the average distance between the positrons in $\mathrm{Ps}_{2}$ is over 6.0 bohr. This indicates that this system is a loosely bound complex of two Ps atoms rather than a molecule with a covalent bond, such as $\mathrm{H}_{2}$. The much smaller binding energy in $\mathrm{Ps}_{2}$ than in $\mathrm{H}_{2}$ confirms this conclusion.

Using the total relativistic energy of the positronium molecule obtained with the largest basis set from Table I and the energy of two noninteracting positronium atoms that include the relativistic corrections, one can compute that the binding energy of $\mathrm{Ps}_{2}$ is 0.01595425 hartree $(0.4341373 \mathrm{eV})$. Even though higher order corrections for the ground state of the positronium atom are known, for the sake of consistency we intentionally did not include them in the above result.

To conclude, accurate calculations of the positronium molecule ground-state nonrelativistic energy and leadingorder relativistic corrections have been carried out using a variational expansion in terms of explicitly correlated Gaussian basis functions. A new upper bound to the nonrelativistic energy has been obtained. The next step in improving the accuracy of the results would be the inclusion of the QED 
corrections proportional to $\alpha^{3}$ and $\alpha^{4}$. It would also be desirable to implement a procedure of regularization (sometimes called "Drachmanization" after Drachman [35]) of the operators whose expectation values converge slowly. Such a procedure works very well in the case of Born-Oppenheimer calculations of atoms and molecules [36] and may also work well for fully nonadiabatic systems, such as the positronium molecule.

This work has been supported by the National Science Foundation. The authors would like to thank University of Arizona Center of Computing and Information Technology for using their supercomputer resources.

\section{APPENDIX: EXPECTATION VALUE OF THE ORBIT- ORBIT INTERACTION OPERATOR}

As we have done in our previous works [29-31], we will keep expressions in matrix form when evaluating the integrals needed in the calculations of the expectation value of the orbit-orbit Hamiltonian, $\hat{H}_{\mathrm{OO}}$. To simplify the notations we will define

$$
\bar{A}=A \otimes I_{3},
$$

where $A$ is a $n \times n$ symmetric matrix of exponential parameters, $I_{3}$ is a $3 \times 3$ identity matrix. With this our explicitly correlated Gaussian basis functions take the following form:

$$
f_{k}=\exp \left(-\mathbf{r}^{\prime} \bar{A}_{k} \mathbf{r}\right)
$$

We will also introduce the following shorthand notation,

$$
\nabla_{\mathbf{r}_{i}} \equiv \nabla_{i}
$$

Greek indices will be used to denote components and summation over repeated greek indices is assumed everywhere below, unless otherwise noted.

\section{Some auxiliary formulas}

There are some simple relations used in deriving the expressions for the integrals, which we would like to list.

(i) By $\boldsymbol{\nabla}_{\mathbf{r}}$ we denote the gradient with respect to the vector of the pseudoparticle coordinates, $\mathbf{r}$, and for $f=\exp \left(-\mathbf{r}^{\prime} \bar{A} \mathbf{r}\right)$ we have

$$
\begin{gathered}
\boldsymbol{\nabla}_{\mathbf{r}}^{\alpha} f \equiv \nabla^{\alpha} f=-2\left(\mathbf{r}^{\prime} \bar{A}\right)^{\alpha} f, \\
\boldsymbol{\nabla}_{\alpha} f=-2(\bar{A} \mathbf{r})_{\alpha} f, \\
\boldsymbol{\nabla}_{\beta} \boldsymbol{\nabla}_{\alpha} f=-2 f \boldsymbol{\nabla}_{\beta}(\bar{A} \mathbf{r})_{\alpha}+(\bar{A} \mathbf{r})_{\alpha} \boldsymbol{\nabla}_{\beta} f \\
=\left[-2 \bar{A}_{\alpha}^{\gamma} \delta_{\beta \gamma}+4(\bar{A} \mathbf{r})_{\alpha}(\bar{A} \mathbf{r})_{\beta}\right] f \\
=\left[4(\bar{A} \mathbf{r})_{\alpha}(\bar{A} \mathbf{r})_{\beta}-2 \bar{A}_{\alpha \beta}\right] f,
\end{gathered}
$$

and

$$
\boldsymbol{\nabla}_{2}^{\beta} \mathbf{r}_{12}^{\alpha}=-\nabla_{2}^{\beta} \mathbf{r}_{2}^{\alpha}=-\delta^{\beta \alpha}
$$

(ii) For $\bar{A}=A \otimes I_{3}$ we have the following relations:

$$
\begin{gathered}
\operatorname{tr}(\bar{A})=3 \operatorname{tr}(A), \\
|\bar{A}|=|A|^{3} .
\end{gathered}
$$

(iii) We define matrix $J_{i j}$ as

$$
J_{i j}= \begin{cases}E_{i i}, & i=j, \\ E_{i i}+E_{j j}-E_{i j}-E_{j i} & i \neq j,\end{cases}
$$

where $E_{i j}$ is the $n \times n$ matrix with 1 in the $i j$ th position and 0 elsewhere. $\left[\left(E_{i j}\right)^{\alpha}{ }_{\beta}=\delta_{i}^{\alpha} \delta_{j \beta}\right]$. It can be easily shown that

$$
\begin{gathered}
E_{i j} E_{i j}=0, \\
E_{i j} E_{j i}=E_{i i}, \\
E_{i j} E_{j j}=E_{i j},
\end{gathered}
$$

when $i \neq j$.

(iv) With the above-defined quantities it can be shown that operators $\boldsymbol{\nabla}_{i}^{\prime} \boldsymbol{\nabla}_{j}$, and $\mathbf{r}_{i j}^{\prime} \boldsymbol{\nabla}_{j}$ can be expressed as follows:

$$
\begin{gathered}
\mathbf{r}_{i j}^{\prime} \boldsymbol{\nabla}_{j}=\left(\mathbf{r}_{i}-\mathbf{r}_{j}\right)^{\prime} \nabla_{j}=\mathbf{r}^{\prime}\left(\bar{E}_{i j}-\bar{E}_{j j}\right) \boldsymbol{\nabla}=\mathbf{r}^{\prime}\left(\bar{E}_{i j}-\bar{E}_{j j}\right) \bar{E}_{j j} \boldsymbol{\nabla}, \\
\boldsymbol{\nabla}_{i}^{\prime} \boldsymbol{\nabla}_{j}=\boldsymbol{\nabla}^{\prime} \bar{E}_{i j} \boldsymbol{\nabla} .
\end{gathered}
$$

Finally, to remove the singularity of the orbit-orbit operator that appears due to the term $1 / r_{i j}^{3}$, we employ the following commuation relation:

$$
\left[\boldsymbol{\nabla}_{i}, \frac{1}{r_{i}}\right]=-\frac{\mathbf{r}_{i}}{r_{i}^{3}},
$$

$$
\left[\boldsymbol{\nabla}_{j}, \frac{1}{r_{i j}}\right]=-\left[\boldsymbol{\nabla}_{i}, \frac{1}{r_{i j}}\right]=\frac{\mathbf{r}_{i j}}{r_{i j}^{3}},
$$

where $\mathbf{r}_{i j}=\mathbf{r}_{i}-\mathbf{r}_{j}$.

\section{Transformation of the orbit-orbit operator}

In the internal coordinate system the orbit-orbit operator has the following form:

$$
\begin{aligned}
\hat{H}_{\mathrm{OO}}(\mathbf{r})= & -\frac{1}{2} \sum_{i=i}^{n} \frac{q_{0} q_{i}}{m_{0} m_{i}}\left(\frac{1}{r_{i}} \boldsymbol{\nabla}_{i}^{\prime} \boldsymbol{\nabla}_{i}+\frac{1}{r_{i}^{3}} \mathbf{r}_{i}^{\prime}\left(\mathbf{r}_{i}^{\prime} \boldsymbol{\nabla}_{i}\right) \boldsymbol{\nabla}_{i}\right) \\
& -\frac{1}{2} \sum_{i=1}^{n} \sum_{j \neq i}^{n} \frac{q_{0} q_{i}}{m_{0} m_{i}}\left(\frac{1}{r_{i}} \boldsymbol{\nabla}_{i}^{\prime} \boldsymbol{\nabla}_{j}+\frac{1}{r_{i}^{3}} \mathbf{r}_{i}^{\prime}\left(\mathbf{r}_{i}^{\prime} \boldsymbol{\nabla}_{i}\right) \boldsymbol{\nabla}_{j}\right) \\
& +\frac{1}{2} \sum_{i=1}^{n-1} \sum_{j \neq i}^{n} \frac{q_{i} q_{j}}{m_{i} m_{j}}\left(\frac{1}{r_{i j}} \boldsymbol{\nabla}_{i}^{\prime} \boldsymbol{\nabla}_{j}+\frac{1}{r_{i j}^{3}} \mathbf{r}_{i j}^{\prime}\left(\mathbf{r}_{i j}^{\prime} \boldsymbol{\nabla}_{i}\right) \boldsymbol{\nabla}_{j}\right) .
\end{aligned}
$$

Using the relations (A1) and (A2) we make the following transformations: 


$$
\begin{gathered}
\frac{1}{r_{i}^{3}} \mathbf{r}_{i}^{\prime}\left(\mathbf{r}_{i}^{\prime} \boldsymbol{\nabla}_{i}\right) \boldsymbol{\nabla}_{j}=\mathbf{r}_{i}^{\prime}\left(\frac{\mathbf{r}_{i}^{\prime}}{r_{i}^{3}} \boldsymbol{\nabla}_{i}\right) \boldsymbol{\nabla}_{j}=r_{i}^{\alpha}\left[\left(-\boldsymbol{\nabla}_{i} \frac{1}{r_{i}}\right) \boldsymbol{\nabla}_{i}\right]\left(\boldsymbol{\nabla}_{j}\right)_{\alpha}, \\
\frac{1}{r_{i j}^{3}} \mathbf{r}_{i j}^{\prime}\left(\mathbf{r}_{i j}^{\prime} \boldsymbol{\nabla}_{i}\right) \boldsymbol{\nabla}_{j}=\mathbf{r}_{i j}^{\prime}\left(\frac{\mathbf{r}_{i j}^{\prime}}{r_{i j}^{3}} \boldsymbol{\nabla}_{i}\right) \boldsymbol{\nabla}_{j}=r_{i j}^{\alpha}\left[\left(\boldsymbol{\nabla}_{j} \frac{1}{r_{i j}}\right) \boldsymbol{\nabla}_{i}\right]\left(\boldsymbol{\nabla}_{j}\right)_{\alpha},
\end{gathered}
$$

which yields

$$
\begin{aligned}
\hat{H}_{\mathrm{OO}}(\mathbf{r})= & -\frac{1}{2} \sum_{i=1}^{n} \frac{q_{0} q_{i}}{m_{0} m_{i}}\left[\frac{1}{r_{i}}\left(\boldsymbol{\nabla}_{i}\right)^{\alpha}\left(\boldsymbol{\nabla}_{i}\right)_{\alpha}-r_{i}^{\alpha}\left(\boldsymbol{\nabla}_{i} \frac{1}{r_{i}}\right)^{\beta}\left(\boldsymbol{\nabla}_{i}\right)_{\beta}\left(\boldsymbol{\nabla}_{i}\right)_{\alpha}\right]-\frac{1}{2} \sum_{i=1}^{n} \sum_{j \neq i}^{n} \frac{q_{0} q_{i}}{m_{0} m_{i}}\left[\frac{1}{r_{i}}\left(\boldsymbol{\nabla}_{i}\right)^{\alpha}\left(\boldsymbol{\nabla}_{j}\right)_{\alpha}-r_{i}^{\alpha}\left(\boldsymbol{\nabla}_{i} \frac{1}{r_{i}}\right)^{\beta}\left(\boldsymbol{\nabla}_{i}\right)_{\beta}\left(\boldsymbol{\nabla}_{j}\right)_{\alpha}\right] \\
& +\frac{1}{2} \sum_{i=1}^{n-1} \sum_{j \neq i}^{n} \frac{q_{i} q_{j}}{m_{i} m_{j}}\left[\frac{1}{r_{i j}}\left(\boldsymbol{\nabla}_{i}\right)^{\alpha}\left(\boldsymbol{\nabla}_{j}\right)_{\alpha}+r_{i j}{ }^{\alpha}\left(\boldsymbol{\nabla}_{j} \frac{1}{r_{i j}}\right)^{\beta}\left(\boldsymbol{\nabla}_{i}\right)_{\beta}\left(\boldsymbol{\nabla}_{j}\right)_{\alpha}\right] .
\end{aligned}
$$

In the next step we rewrite the orbit-orbit operator using the above-described matrix notation,

$$
\begin{aligned}
\hat{H}_{\mathrm{OO}}(r)= & -\frac{1}{2} \sum_{i=1}^{n} \frac{q_{0} q_{i}}{m_{0} m_{i}}\left[\frac{1}{r_{i}}\left(\boldsymbol{\nabla}^{\prime} \bar{E}_{i i} \boldsymbol{\nabla}\right)\right. \\
& \left.-\left(\mathbf{r}^{\prime} \bar{E}_{i i}\right)^{\alpha}\left(\boldsymbol{\nabla}^{\prime} \bar{E}_{i i} \frac{1}{r_{i}}\right)^{\beta}\left(\bar{E}_{i i} \boldsymbol{\nabla}\right)_{\beta}\left(\bar{E}_{i i} \boldsymbol{\nabla}\right)_{\alpha}\right] \\
& -\frac{1}{2} \sum_{i=1}^{n} \sum_{j \neq i}^{n} \frac{q_{0} q_{i}}{m_{0} m_{i}}\left[\frac{1}{r_{i}}\left(\boldsymbol{\nabla}^{\prime} \bar{E}_{i j} \boldsymbol{\nabla}\right)\right. \\
& \left.-\left(\mathbf{r}^{\prime} \bar{E}_{i i}\right)^{\alpha}\left(\boldsymbol{\nabla}^{\prime} \bar{E}_{i i} \frac{1}{r_{i}}\right)^{\beta}\left(\bar{E}_{i i} \boldsymbol{\nabla}\right)_{\beta}\left(\bar{E}_{i j} \boldsymbol{\nabla}\right)_{\alpha}\right] \\
& +\frac{1}{2} \sum_{i=1}^{n-1} \sum_{j \neq i}^{n} \frac{q_{i} q_{j}}{m_{i} m_{j}}\left[\frac{1}{r_{i j}}\left(\nabla^{\prime} \bar{E}_{i j} \boldsymbol{\nabla}\right)+\left[\mathbf { r } ^ { \prime } \left(\bar{E}_{i j}\right.\right.\right. \\
& \left.\left.\left.-\bar{E}_{j j}\right)\right]^{\alpha}\left(\boldsymbol{\nabla}^{\prime} \bar{E}_{j i} \frac{1}{r_{i j}}\right)^{\beta}\left(\bar{E}_{i i} \boldsymbol{\nabla}\right)_{\beta}\left(\bar{E}_{j j} \boldsymbol{\nabla}\right)_{\alpha}\right] .
\end{aligned}
$$

To simplify the expression for the expectation value of the $\hat{H}_{\mathrm{OO}}(\mathbf{r})$ operator we use the following general integral for each of the three terms that appear in the expectation value,

$$
\left\langle f_{k}\left|\left[\frac{1}{r_{g}}\left(\boldsymbol{\nabla}^{\prime} \bar{B} \boldsymbol{\nabla}\right)-\left(\mathbf{r}^{\prime} \bar{C}\right)^{\alpha}\left(\boldsymbol{\nabla}^{\prime} \bar{D} \frac{1}{r_{g}}\right)^{\beta}(\bar{F} \boldsymbol{\nabla})_{\beta}(\bar{G} \boldsymbol{\nabla})_{\alpha}\right]\right| f_{l}\right\rangle,
$$

for term (A3a): $g=i, \quad \bar{B}=\bar{E}_{i i}, \quad \bar{C}=\bar{E}_{i i}, \quad \bar{D}=\bar{E}_{i i}$,

$$
\bar{F}=\bar{E}_{i i}, \quad \bar{G}=\bar{E}_{i i}
$$

for term (A3b): $g=i, \quad \bar{B}=\bar{E}_{i j}, \quad \bar{C}=\bar{E}_{i i}, \quad \bar{D}=\bar{E}_{i i}$,

$$
\bar{F}=\bar{E}_{i i}, \quad \bar{G}=\bar{E}_{i j} ;
$$

for term (A3c): $g=i j, \quad \bar{B}=\bar{E}_{i j}, \quad \bar{C}=\left(\bar{E}_{i j}-\bar{E}_{j j}\right)$,

$$
\bar{D}=\bar{E}_{j i}, \quad \bar{F}=\bar{E}_{i i}, \quad \bar{G}=\bar{E}_{j j}
$$

Hence, in order to calculate the expectation value of $\hat{H}_{\mathrm{OO}}(\mathbf{r})$ we need to evaluate the following integrals:

$$
\begin{gathered}
\left\langle f_{k}\left|\frac{1}{r_{g}}\left(\nabla^{\prime} \bar{B} \boldsymbol{\nabla}\right)\right| f_{l}\right\rangle, \\
\left\langle f_{k}\left|\left(\mathbf{r}^{\prime} \bar{C}\right)^{\alpha}\left(\boldsymbol{\nabla}^{\prime} \bar{D} \frac{1}{r_{g}}\right)^{\beta}(\bar{F} \boldsymbol{\nabla})_{\beta}(\bar{G} \boldsymbol{\nabla})_{\alpha}\right| f_{l}\right\rangle .
\end{gathered}
$$

Next we will derive the formulas for the two integrals.

\section{Integral $\left\langle f_{k}\left|\frac{1}{r_{g}}\left(\nabla^{\prime} \bar{B} \nabla\right)\right| f_{l}\right\rangle$}

First we apply the operator $\nabla^{\prime} \bar{B} \boldsymbol{\nabla}$ to $f_{l}$,

$$
\begin{aligned}
\boldsymbol{\nabla}^{\prime} \bar{B} \boldsymbol{\nabla} f_{l} & =\nabla^{\alpha}(\bar{B})_{\alpha}{ }^{\beta} \boldsymbol{\nabla}_{\beta} f_{l}=\bar{B}_{\alpha}{ }^{\beta} \nabla^{\alpha} \nabla_{\beta} f_{l} \\
& =\bar{B}_{\alpha}{ }^{\beta}\left[4\left(\mathbf{r}^{\prime} \bar{A}_{l}\right)^{\alpha}\left(\bar{A}_{l} \mathbf{r}\right)_{\beta}-2\left(\bar{A}_{l}\right)_{\beta}{ }^{\alpha}\right] f_{l} \\
& =4\left[\left(\mathbf{r}^{\prime} \bar{A}_{l}\right)^{\alpha} \bar{B}_{\alpha}{ }^{\beta}\left(\bar{A}_{l} \mathbf{r}\right)_{\beta}-2 \bar{B}_{\alpha}{ }^{\beta}\left(\bar{A}_{l}\right)_{\beta}{ }^{\alpha}\right] f_{l} \\
& =4\left(\mathbf{r}^{\prime} \bar{A}_{l} \bar{B} \bar{A}_{l} \mathbf{r}\right) f_{l}-6 \operatorname{tr}\left(B A_{l}\right) f_{l} .
\end{aligned}
$$

With that we can now calculate the value of the integral,

$$
\left\langle f_{k}\left|\frac{1}{r_{g}} \nabla^{\prime} \bar{B} \boldsymbol{\nabla}\right| f_{l}\right\rangle=4\left\langle f_{k}\left|\frac{1}{r_{g}}\left(\mathbf{r}^{\prime} \bar{A}_{l} \bar{B} \bar{A}_{l} \mathbf{r}\right)\right| f_{l}\right\rangle-6 \operatorname{tr}\left(B A_{l}\right)\left\langle f_{k}\left|\frac{1}{r_{g}}\right| f_{l}\right\rangle .
$$

\section{Integral $\left\langle f_{k}\left|\left(\mathbf{r}^{\prime} \bar{C}\right)^{\alpha}\left(\nabla^{\prime} \bar{D} \frac{1}{r_{g}}\right)^{\beta}(\bar{F} \nabla)_{\beta}(\bar{G} \nabla)_{\alpha}\right| f_{l}\right\rangle$}

We rewrite the integral in the following way:

$$
\begin{aligned}
& \left\langle f_{k}\left|\left(\mathbf{r}^{\prime} \bar{C}\right)^{\alpha}\left(\boldsymbol{\nabla}^{\prime} \bar{D} \frac{1}{r_{g}}\right)^{\beta}(\bar{F} \boldsymbol{\nabla})_{\beta}(\bar{G} \boldsymbol{\nabla})_{\alpha}\right| f_{l}\right\rangle \\
& =-\int d \tau \frac{1}{r_{g}}\left(\nabla^{\prime} \bar{D}\right)^{\beta}\left[f_{k}\left(\mathbf{r}^{\prime} \bar{C}\right)^{\alpha}(\bar{F} \boldsymbol{\nabla})_{\beta}(\bar{G} \boldsymbol{\nabla})_{\alpha} f_{l}\right],
\end{aligned}
$$

where the operator in the integral can be split into three terms: 


$$
\begin{aligned}
\left(\boldsymbol{\nabla}^{\prime} \bar{D}\right)^{\beta} & {\left[f_{k}\left(\mathbf{r}^{\prime} \bar{C}\right)^{\alpha}(\bar{F} \boldsymbol{\nabla})_{\beta}(\bar{G} \boldsymbol{\nabla})_{\alpha} f_{l}\right] } \\
= & {\left[\left(\boldsymbol{\nabla}^{\prime} \bar{D}\right)^{\beta} f_{k}\right]\left(\mathbf{r}^{\prime} \bar{C} \bar{G} \boldsymbol{\nabla}\right)(\bar{F} \boldsymbol{\nabla})_{\beta} f_{l} \quad \text { term } 1 } \\
& +f_{k}\left[\left(\boldsymbol{\nabla}^{\prime} \bar{D}\right)^{\beta}\left(\mathbf{r}^{\prime} \bar{C}\right)^{\alpha}\right](\bar{F} \boldsymbol{\nabla})_{\beta}(\bar{G} \boldsymbol{\nabla})_{\alpha} f_{l} \quad \text { term } 2 \\
& +f_{k}\left(\mathbf{r}^{\prime} \bar{C}\right)^{\alpha}\left[\left(\boldsymbol{\nabla}^{\prime} \bar{D}\right)^{\beta}(\bar{F} \boldsymbol{\nabla})_{\beta}(\bar{G} \boldsymbol{\nabla})_{\alpha} f_{l}\right] \text { term 3, }
\end{aligned}
$$

and now we evaluate each term separately.

\section{a. Term 1: $\left[\left(\nabla^{\prime} \bar{D}\right)^{\beta} f_{k}\right]\left(\mathbf{r}^{\prime} \bar{C} \bar{G} \nabla\right)(\bar{F} \nabla)_{\beta} f_{l}$}

First we make the following transformations:

$$
\left(\nabla^{\prime} \bar{D}\right)^{\beta} f_{k}=\bar{D}_{\gamma}^{\beta} \nabla^{\gamma} f_{k}=-2\left(\mathbf{r}^{\prime} \bar{A}_{k} \bar{D}\right)^{\beta} f_{k},
$$

$\left(\mathbf{r}^{\prime} \bar{C} \bar{G} \boldsymbol{\nabla}\right)(\bar{F} \boldsymbol{\nabla})_{\beta} f_{l}=\left[4\left(\mathbf{r}^{\prime} \bar{C} \bar{G} \bar{A}_{l} \mathbf{r}\right)\left(\bar{F} \bar{A}_{l} \mathbf{r}\right)_{\beta}-2\left(\bar{F} \bar{A}_{l} \bar{G} \bar{C} \mathbf{r}\right)_{\beta}\right] f_{l}$.

The first of the above transformations can be easily derived and the second results from the following:

$$
\begin{aligned}
\left(\mathbf{r}^{\prime} \bar{C} \bar{G}\right)^{\alpha} \bar{F}_{\beta}{ }^{\rho} \nabla_{\alpha} \nabla_{\rho} f_{l}= & \left(\mathbf{r}^{\prime} \bar{C} \bar{G}\right)^{\alpha} \bar{F}_{\beta}{ }^{\rho}\left[4\left(\overline{A_{l}} \mathbf{r}\right)_{\alpha}\left(\overline{A_{l}} \mathbf{r}\right)_{\rho}-2\left(\bar{A}_{l}\right)_{\alpha \rho}\right] f_{l} \\
= & {\left[4\left(\mathbf{r}^{\prime} \bar{C} \bar{G}\right)^{\alpha}\left(\bar{A}_{l} \mathbf{r}\right)_{\alpha} \bar{F}_{\beta}{ }^{\rho}\left(\bar{A}_{l} \mathbf{r}\right)_{\rho}\right.} \\
& \left.-2 \bar{F}_{\beta}{ }^{\rho}\left(\bar{A}_{l}\right)_{\alpha \rho}(\bar{G} \bar{C} \mathbf{r})^{\alpha}\right] f_{l} \\
= & {\left[4\left(\mathbf{r}^{\prime} \bar{C} \bar{G} \bar{A}_{l} \mathbf{r}\right)\left(\bar{F} \bar{A}_{l} \mathbf{r}\right)_{\beta}-2\left(\bar{F} \bar{A}_{l} \bar{G} \bar{C} \mathbf{r}\right)_{\beta}\right] f_{l} . }
\end{aligned}
$$

With that, the expectation value of term 1 can now be evaluated as

$$
\begin{gathered}
\int d \tau \frac{1}{r_{g}}\left[\left(\boldsymbol{\nabla}^{\prime} \bar{D}\right)^{\beta} f_{k}\right]\left(\mathbf{r}^{\prime} \bar{C} \bar{G} \boldsymbol{\nabla}\right)(\bar{F} \boldsymbol{\nabla})_{\beta} f_{l} \\
=-8\left\langle f_{k}\left|\frac{1}{r_{g}}\left(\mathbf{r}^{\prime} \bar{C} \bar{G} \bar{A}_{l} \mathbf{r}\right)\left(\mathbf{r}^{\prime} \bar{A}_{k} \bar{D} \bar{F} \bar{A}_{l} \mathbf{r}\right)\right| f_{l}\right\rangle \\
+4\left\langle f_{k}\left|\frac{1}{r_{g}}\left(\mathbf{r}^{\prime} \bar{A}_{k} \bar{D} \bar{F} \bar{A}_{l} \bar{G} \bar{C} \mathbf{r}\right)\right| f_{l}\right\rangle .
\end{gathered}
$$

\section{b. Term 2: $f_{k}\left[\left(\nabla^{\prime} \bar{D}\right)^{\beta}\left(\mathbf{r}^{\prime} \bar{C}\right)^{\alpha}\right](\bar{F} \nabla)_{\beta}(\bar{G} \nabla)_{\alpha} f_{l}$}

Here we make use of the following relations:

$$
\begin{gathered}
\left(\nabla^{\prime} \bar{D}\right)^{\beta}\left(\mathbf{r}^{\prime} \bar{C}\right)^{\alpha}=\bar{D}_{\gamma}{ }_{\gamma} \bar{C}^{\gamma \alpha}, \\
{\left[\left(\nabla^{\prime} \bar{D}\right)^{\beta}\left(\mathbf{r}^{\prime} \bar{C}\right)^{\alpha}\right](\bar{F} \boldsymbol{\nabla})_{\beta}(\bar{G} \boldsymbol{\nabla})_{\alpha} f_{l}=\left(\nabla^{\prime} \bar{G} \bar{C} \bar{D} \bar{F} \boldsymbol{\nabla}\right) f_{l} .}
\end{gathered}
$$

The first of those relations is obtained as follows:

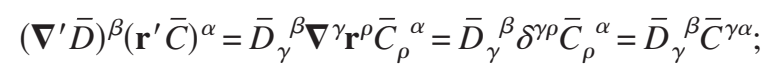

and the second one results from the following transformations:

$$
\begin{aligned}
\left(\bar{D}_{\gamma}{ }^{\beta} \bar{C}^{\gamma \alpha}\right)(\bar{F} \boldsymbol{\nabla})_{\beta}(\bar{G} \boldsymbol{\nabla})_{\alpha} f_{l} & =\bar{D}_{\gamma}^{\beta}(\bar{F} \boldsymbol{\nabla})_{\beta}(\bar{C} \bar{G} \boldsymbol{\nabla})^{\gamma} f_{l} \\
& =\left(\boldsymbol{\nabla}^{\prime} \bar{G} \bar{C}\right)^{\gamma} \overline{D_{\gamma}}{ }_{\gamma}^{\beta}(\bar{F} \boldsymbol{\nabla})_{\beta} f_{l} \\
& =\left(\boldsymbol{\nabla}^{\prime} \bar{G} \bar{C} \bar{D} \bar{F} \boldsymbol{\nabla}\right) f_{l} .
\end{aligned}
$$

From here, analogously to the expression obtained in (A4), we have

$$
\left(\boldsymbol{\nabla}^{\prime} \bar{M} \boldsymbol{\nabla}\right) f_{l}=4\left(\mathbf{r}^{\prime} \bar{A}_{l} \bar{M} \bar{A}_{l} \mathbf{r}\right) f_{l}-6 \operatorname{tr}\left(M A_{l}\right) f_{l} .
$$

Now, we can evaluate the expectation value of term 2 as

$$
\begin{aligned}
& \left\langle f_{k}\left|\frac{1}{r_{g}}\left[\left(\nabla^{\prime} \bar{D}\right)^{\beta}\left(\mathbf{r}^{\prime} \bar{C}\right)^{\alpha}\right](\bar{F} \boldsymbol{\nabla})_{\beta}(\bar{G} \boldsymbol{\nabla})_{\alpha}\right| f_{l}\right\rangle \\
& \quad=4\left\langle f_{k}\left|\frac{1}{r_{g}}\left(\mathbf{r}^{\prime} \overline{A_{l}} \bar{G} \bar{C} \bar{D} \bar{F} \bar{A}_{l} \mathbf{r}\right)\right| f_{l}\right\rangle-6 \operatorname{tr}\left(G^{\prime} C^{\prime} D F A_{l}\right)\left\langle f_{k}\left|\frac{1}{r_{g}}\right| f_{l}\right\rangle .
\end{aligned}
$$

\section{c. Term 3: $f_{k}\left(\mathbf{r}^{\prime} \bar{C}\right)^{\alpha}\left[\left(\nabla^{\prime} \bar{D}\right)^{\beta}(\bar{F} \nabla)_{\beta}(\bar{G} \nabla)_{\alpha} f_{l}\right]$}

Here we use the following relations:

$$
\begin{gathered}
\left(\mathbf{r}^{\prime} \bar{C}\right)^{\alpha}\left[\left(\boldsymbol{\nabla}^{\prime} \bar{D}\right)^{\beta}(\bar{F} \boldsymbol{\nabla})_{\beta}(\bar{G} \boldsymbol{\nabla})_{\alpha} f_{l}\right] \\
\quad=\left(\mathbf{r}^{\prime} \bar{C} \bar{G}\right)^{\alpha}(\bar{D} \bar{F})_{\rho}^{\beta} \boldsymbol{\nabla}^{\rho} \nabla_{\alpha} \nabla_{\beta} f_{l} ;
\end{gathered}
$$

which results from the following simple transformations:

$$
\begin{aligned}
\left(\mathbf{r}^{\prime} \bar{C}\right)^{\alpha}(\bar{G} \boldsymbol{\nabla})_{\alpha}\left(\boldsymbol{\nabla}^{\prime} \bar{D}\right)^{\beta}(\bar{F} \boldsymbol{\nabla})_{\beta} f_{l}=\left(\mathbf{r}^{\prime} \bar{C} \bar{G} \boldsymbol{\nabla}\right)\left(\boldsymbol{\nabla}^{\prime} \bar{D} \bar{F} \boldsymbol{\nabla}\right) f_{l} \\
=\left(\mathbf{r}^{\prime} \bar{C} \bar{G}\right)^{\alpha}(\bar{D} \bar{F})_{\rho}{ }^{\beta} \boldsymbol{\nabla}^{\rho} \nabla_{\alpha} \nabla_{\beta} f_{l}, \\
\boldsymbol{\nabla}^{\rho} \nabla_{\alpha} \nabla_{\beta} f_{l}=4\left[\left(\bar{A}_{l}\right)_{\alpha}{ }^{\rho}\left(\bar{A}_{l} \mathbf{r}\right)_{\beta}+\left(\bar{A}_{l} \mathbf{r}\right)_{\alpha}\left(\bar{A}_{l}\right)_{\beta}{ }^{\rho}\right] f_{l} \\
-4\left[2\left(\mathbf{r}^{\prime} \bar{A}_{l}\right)^{\rho}\left(\bar{A}_{l} \mathbf{r}\right)_{\alpha}\left(\bar{A}_{l} \mathbf{r}\right)_{\beta}-\left(\mathbf{r}^{\prime} \bar{A}_{l}\right)^{\rho}\left(\bar{A}_{l}\right)_{\alpha \beta}\right] f_{l} ;
\end{aligned}
$$

which results from the following transformations:

$$
\begin{aligned}
\nabla^{\rho} \nabla_{\alpha} \nabla_{\beta} f_{l}= & \nabla^{\rho}\left\{\left[4\left(\bar{A}_{l} \mathbf{r}\right)_{\alpha}\left(\bar{A}_{l} \mathbf{r}\right)_{\beta}-2\left(\bar{A}_{l}\right)_{\alpha \beta}\right] f_{l}\right\} \\
= & \nabla^{\rho}\left\{\left[4\left(\bar{A}_{l}\right)_{\alpha}{ }^{\gamma} \mathbf{r}_{\gamma}\left(\bar{A}_{l}\right)_{\beta}{ }^{\xi} \mathbf{r}_{\xi}-2\left(\bar{A}_{l}\right)_{\alpha \beta}\right] f_{l}\right\} \\
= & 4\left[\left(\bar{A}_{l}\right)_{\alpha}^{\gamma} \delta_{\rho}^{\gamma}\left(\bar{A}_{l} \mathbf{r}\right)_{\beta}+\left(\bar{A}_{l} \mathbf{r}\right)_{\alpha}\left(\bar{A}_{l}\right)_{\beta}{ }^{\xi} \delta_{\rho}{ }^{\xi}\right] f_{l} \\
& -4\left[2\left(\bar{A}_{l} \mathbf{r}\right)_{\alpha}\left(\bar{A}_{l} \mathbf{r}\right)_{\beta}-\left(\bar{A}_{l}\right)_{\alpha \beta}\right]\left(\mathbf{r}^{\prime} \bar{A}_{l}\right)^{\rho} f_{l} \\
= & 4\left[\left(\bar{A}_{l}\right)_{\alpha}{ }^{\rho}\left(\bar{A}_{l} \mathbf{r}\right)_{\beta}+\left(\bar{A}_{l} \mathbf{r}\right)_{\alpha}\left(\bar{A}_{l}\right)_{\beta}{ }^{\rho}\right] f_{l} \\
& -4\left[2\left(\mathbf{r}^{\prime} \bar{A}_{l}\right)^{\rho}\left(\bar{A}_{l} \mathbf{r}\right)_{\alpha}\left(\bar{A}_{l} \mathbf{r}\right)_{\beta}-\left(\mathbf{r}^{\prime} \bar{A}_{l}\right)^{\rho}\left(\bar{A}_{l}\right)_{\alpha \beta}\right] f_{l},
\end{aligned}
$$

$$
\begin{aligned}
\left(\mathbf{r}^{\prime} \bar{C}\right)^{\alpha} & \left(\nabla^{\prime} \bar{D}\right)^{\beta}(\bar{F} \boldsymbol{\nabla})_{\beta}(\bar{G} \boldsymbol{\nabla})_{\alpha} f_{l} \\
= & 4\left(\mathbf{r}^{\prime} \bar{C} \bar{G} \bar{A} \bar{D} \bar{D} \overline{A_{l}} \mathbf{r}\right) f_{l}+4\left(\mathbf{r}^{\prime} \bar{C} \bar{G} \bar{A}_{l} \mathbf{r}\right) \operatorname{tr}\left(\bar{D} \bar{F} \bar{A}_{l}\right) f_{l} \\
& -8\left(\mathbf{r}^{\prime} \bar{C} \bar{G} \bar{A}_{l} \mathbf{r}\right)\left(\mathbf{r}^{\prime} \overline{A_{l}} \bar{D} \bar{F} \bar{A}_{l} \mathbf{r}\right) f_{l}+4\left(\mathbf{r}^{\prime} \overline{A_{l}} \bar{D} \bar{F} \bar{A}_{l} \bar{G} \bar{C} \mathbf{r}\right) f_{l} ;
\end{aligned}
$$

which is obtained as follows: 


$$
\begin{aligned}
& \left(\mathbf{r}^{\prime} \bar{C}\right)^{\alpha}\left(\boldsymbol{\nabla}^{\prime} \bar{D}\right)^{\beta}(\bar{F} \boldsymbol{\nabla})_{\beta}(\bar{G} \boldsymbol{\nabla})_{\alpha} f_{l}=\left(\mathbf{r}^{\prime} \bar{C} \bar{G}\right)^{\alpha}(\bar{D} \bar{F})_{\rho}^{\beta} \boldsymbol{\nabla}^{\rho} \nabla_{\alpha} \nabla_{\beta} f_{l} \\
& =4\left(\mathbf{r}^{\prime} \bar{C} \bar{G}\right)^{\alpha}(\bar{D} \bar{F})_{\rho}{ }^{\beta}\left[\left(\bar{A}_{l}\right)_{\alpha}{ }^{\rho}\left(\bar{A}_{l} \mathbf{r}\right)_{\beta}+\left(\bar{A}_{l} \mathbf{r}\right)_{\alpha}\left(\bar{A}_{l}\right)_{\beta}{ }^{\rho}\right] f_{l}+\left(\mathbf{r}^{\prime} \bar{C} \bar{G}\right)^{\alpha}(\bar{D} \bar{F})_{\rho}^{\beta}\left[-8\left(\mathbf{r}^{\prime} \bar{A}_{l}\right)^{\rho}\left(\bar{A}_{l} \mathbf{r}\right)_{\alpha}\left(\overline{A_{l}} \mathbf{r}\right)_{\beta}\right. \\
& \left.+4\left(\mathbf{r}^{\prime} \bar{A}_{l}\right)^{\rho}\left(\bar{A}_{l}\right)_{\alpha \beta}\right] f_{l} \\
& =4\left(\mathbf{r}^{\prime} \bar{C} \bar{G}\right)^{\alpha}\left[\left(\bar{A}_{l}\right)_{\alpha}{ }^{\rho}(\bar{D} \bar{F})_{\rho}{ }^{\beta}\left(\bar{A}_{l} \mathbf{r}\right)_{\beta}+\left(\bar{A}_{l} \mathbf{r}\right)_{\alpha}(\bar{D} \bar{F})_{\rho}{ }^{\beta}\left(\bar{A}_{l}\right)_{\beta}{ }^{\rho}\right] f_{l} \\
& -8\left(\mathbf{r}^{\prime} \bar{C} \bar{G}\right)^{\alpha}\left(\bar{A}_{l} \mathbf{r}\right)_{\alpha}\left(\mathbf{r}^{\prime} \bar{A}_{l}\right)^{\rho}(\bar{D} \bar{F})_{\rho}{ }^{\beta}\left(\bar{A}_{l} \mathbf{r}\right)_{\beta} f_{l}+4\left(\mathbf{r}^{\prime} \bar{A}_{l}\right)^{\rho}(\bar{D} \bar{F})_{\rho}{ }^{\beta}\left(\bar{A}_{l}\right)_{\alpha \beta}\left(\mathbf{r}^{\prime} \bar{C} \bar{G}\right)^{\alpha} f_{l} \\
& =4\left(\mathbf{r}^{\prime} \bar{C} \bar{G} \bar{A}_{l} \bar{D} \bar{F} \bar{A}_{l} \mathbf{r}\right) f_{l}+4\left(\mathbf{r}^{\prime} \bar{C} \bar{G} \bar{A}_{l} \mathbf{r}\right) \operatorname{tr}\left(\bar{D} \bar{F} \bar{A}_{l}\right) f_{l}-8\left(\mathbf{r}^{\prime} \bar{C} \bar{G} \bar{A}_{l} \mathbf{r}\right)\left(\mathbf{r}^{\prime} \bar{A}_{l} \bar{D} \bar{F} \bar{A}_{l} \mathbf{r}\right) f_{l} \\
& +4\left(\mathbf{r}^{\prime} \overline{A_{l}} \bar{D} \bar{F} \overline{A_{l}} \bar{G} \bar{C} \mathbf{r}\right) f_{l} \text {. }
\end{aligned}
$$

The above three relations allow us to evaluate the expectation value of the operator in term 3 as

$$
\begin{aligned}
\left\langle f_{k}\left|\frac{1}{r_{g}}\left(\mathbf{r}^{\prime} \bar{C}\right)^{\alpha}\left(\boldsymbol{\nabla}^{\prime} \bar{D}\right)^{\beta}(\bar{F} \boldsymbol{\nabla})_{\beta}(\bar{G} \boldsymbol{\nabla})_{\alpha}\right| f_{l}\right\rangle= & 4\left\langle f_{k}\left|\frac{1}{r_{g}}\left(\mathbf{r}^{\prime} \bar{C} \bar{G} \overline{A_{l}} \bar{D} \bar{F} \bar{A}_{l} \mathbf{r}\right)\right| f_{l}\right\rangle+4\left\langle f_{k}\left|\frac{1}{r_{g}}\left(\mathbf{r}^{\prime} \bar{A}_{l} \bar{D} \bar{F} \bar{A}_{l} \bar{G} \bar{C} \mathbf{r}\right)\right| f_{l}\right\rangle-8\left\langle f_{k}\right| \frac{1}{r_{g}}\left(\mathbf{r}^{\prime} \bar{C} \bar{G} \overline{A_{l}} \mathbf{r}\right) \\
& \times\left(\mathbf{r}^{\prime} \bar{A}_{l} \bar{D} \bar{F} \bar{A} \bar{A}_{l} \mathbf{r}\right)\left|f_{l}\right\rangle+12 \operatorname{tr}\left(D F A_{l}\right)\left\langle f_{k}\left|\frac{1}{r_{g}}\left(\mathbf{r}^{\prime} \bar{C} \bar{G} \bar{A}_{l} \mathbf{r}\right)\right| f_{l}\right\rangle .
\end{aligned}
$$

Now collecting the terms from (A7)-(A9) we determine the expectation value (A5) as

$$
\begin{aligned}
\left\langle f_{k}\left|\frac{1}{r_{g}}\left(\mathbf{r}^{\prime} \bar{C}\right)^{\alpha}\left(\nabla^{\prime} \bar{D} \frac{1}{r_{g}}\right)^{\beta}(\bar{F} \boldsymbol{\nabla})_{\beta}(\bar{G} \boldsymbol{\nabla})_{\alpha}\right| f_{l}\right\rangle= & -4\left\langle f_{k}\left|\frac{1}{r_{g}}\left(\mathbf{r}^{\prime} \overline{A_{l}} \bar{G} \bar{C} \bar{D} \bar{F} \bar{A}_{l} \mathbf{r}\right)\right| f_{l}\right\rangle-4\left\langle f_{k}\left|\frac{1}{r_{g}}\left(\mathbf{r}^{\prime} \bar{C} \bar{G} \overline{A_{l}} \bar{D} \bar{F} \overline{A_{l}} \mathbf{r}\right)\right| f_{l}\right\rangle-4\left\langle f_{k}\right| \frac{1}{r_{g}}\left(\mathbf{r}^{\prime} \bar{A}_{l} \bar{D} \bar{F} \overline{A_{l}} \bar{G} \bar{C} \mathbf{r}\right) \\
& \times\left|f_{l}\right\rangle-4\left\langle f_{k}\left|\frac{1}{r_{g}}\left(\mathbf{r}^{\prime} \bar{A}_{k} \bar{D} \bar{F} \overline{A_{l}} \bar{G} \bar{C} \mathbf{r}\right)\right| f_{l}\right\rangle+6 \operatorname{tr}\left(G^{\prime} C^{\prime} D F A_{l}\right)\left\langle f_{k}\left|\frac{1}{r_{g}}\right| f_{l}\right\rangle-12 \operatorname{tr}\left(D F A_{l}\right) \\
& \times\left\langle f_{k}\left|\frac{1}{r_{g}}\left(\mathbf{r}^{\prime} \bar{C} \bar{G} \bar{A}_{l} \mathbf{r}\right)\right| f_{l}\right\rangle+8\left\langle f_{k}\left|\frac{1}{r_{g}}\left(\mathbf{r}^{\prime} \bar{C} \bar{G} \bar{A}_{l} \mathbf{r}\right)\left(\mathbf{r}^{\prime} \overline{A_{l}} \bar{D} \bar{F} \bar{A}_{l} \mathbf{r}\right)\right| f_{l}\right\rangle+8\left\langle f_{k}\right| \frac{1}{r_{g}}\left(\mathbf{r}^{\prime} \bar{C} \bar{G} \overline{A_{l}} \mathbf{r}\right) \\
& \times\left(\mathbf{r}^{\prime} \bar{A}_{k} \bar{D} \bar{F} \overline{A_{l}} \mathbf{r}\right)\left|f_{l}\right\rangle .
\end{aligned}
$$

Having evaluated the general integrals (A4) and (A5) we have all of the expressions needed to determine the expectation value of the $\hat{H}_{\mathrm{OO}}(\mathbf{r})$ operator.

[1] J. A. Wheeler, Ann. N.Y. Acad. Sci. 48, 219 (1946).

[2] A. P. Mills, Jr., Phys. Rev. Lett. 50, 671 (1983).

[3] A. K. Bhatia and R. J. Drachman, Nucl. Instrum. Methods Phys. Res. B 143, 195 (1998).

[4] G. W. F. Drake and M. Grigorescu, J. Phys. B 38, 3377 (2005).

[5] E. A. Hylleraas and A. Ore, Phys. Rev. 71, 493 (1947).

[6] D. B. Cassidy, S. H. M. Deng, R. G. Greaves, and A. P. Mills, Jr., Rev. Sci. Instrum. 77, 073106 (2006).

[7] A. P. Mills, Jr., Nucl. Instrum. Methods Phys. Res. B 192, 107 (2002).

[8] Y. K. Ho, Phys. Rev. A 33, 3584 (1986).

[9] A. M. Frolov, S. I. Kryuchkov, and V. H. Smith, Phys. Rev. A 51, 4514 (1995).

[10] T. K. Rebane, V. S. Zotev, and O. N. Yusupov, Zh. Eksp. Teor. Fiz. 110, 55 (1996).

[11] A. M. Frolov and V. H. Smith, Phys. Rev. A 55, 2662 (1997).

[12] J. Usukura, K. Varga, and Y. Suzuki, Phys. Rev. A 58, 1918 (1998).

[13] D. H. Bailey and A. M. Frolov, Phys. Rev. A 72, 014501 (2005).
[14] S. Bubin and L. Adamowicz, Phys. Rev. A 74, 052502 (2006).

[15] K. Varga, J. Usukura, and Y. Suzuki, Phys. Rev. Lett. 80, 1876 (1998).

[16] D. B. Kinghorn and R. D. Poshusta, Phys. Rev. A 47, 3671 (1993).

[17] P. M. Kozlowski and L. Adamowicz, Phys. Rev. A 48, 1903 (1993).

[18] D. Bressanini, M. Mella, and G. Morosi, Phys. Rev. A 55, 200 (1997).

[19] Z.-C. Yan and G. W. F. Drake, Phys. Rev. Lett. 81, 774 (1998).

[20] Z.-C. Yan and G. W. F. Drake, Phys. Rev. Lett. 91, 113004 (2003).

[21] D. C. Morton, Q. Wu, and G. W. F. Drake, Can. J. Phys. 84, 83 (2006).

[22] K. Pachucki and J. Komasa, Phys. Rev. Lett. 92, 213001 (2004).

[23] M. Puchalski, A. M. Moro, and K. Pachucki, Phys. Rev. Lett. 97, 133001 (2006).

[24] W. Cencek, J. Komasa, K. Pachucki, and K. Szalewicz, Phys. Rev. Lett. 95, 233004 (2005).

[25] M. Stanke, D. Kȩdziera, M. Molski, S. Bubin, M. Barysz, and 
L. Adamowicz, Phys. Rev. Lett. 96, 233002 (2006).

[26] Z.-C. Yan and Y. K. Ho, Phys. Rev. A 60, 5098 (1999).

[27] H. A. Bethe and E. E. Salpeter, Quantum Mechanics of Oneand Two-Electron Atoms (Plenum, New York, 1977).

[28] V. B. Berestetskii, E. M. Lifshitz, and L. P. Pitaevskii, Quantum Electrodynamics (Nauka, Moscow, 1989) (in Russian).

[29] S. Bubin and L. Adamowicz, J. Chem. Phys. 124, 224317 (2006).

[30] S. Bubin, M. Cafiero, and L. Adamowicz, Adv. Chem. Phys. 131, 377 (2005).
[31] D. Kȩdziera, M. Stanke, S. Bubin, M. Barysz, and L. Adamowicz, J. Chem. Phys. 125, 084303 (2006).

[32] M. Stanke, D. Kędziera, S. Bubin, and L. Adamowicz, J. Chem. Phys. 126, 194312 (2007).

[33] D. M. Schrader, Phys. Rev. Lett. 92, 043401 (2004).

[34] D. M. Schrader, Nucl. Instrum. Methods Phys. Res. B 221, 182 (2004).

[35] R. J. Drachman, J. Phys. B 14, 2733 (1981).

[36] K. Pachucki, W. Cencek, and J. Komasa, J. Chem. Phys. 122, 184101 (2005). 